Kompass

Pneumologie

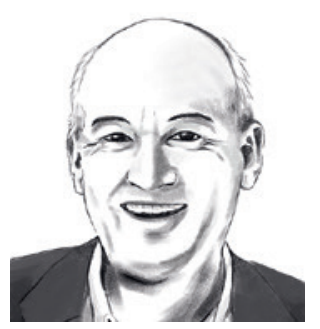

Harald Walach

Poznan/Berlin

Polen/Deutschland

Jetzt ist das Ei rehabilitiert. Endlich, nach vielleicht 5 Dekaden wird aufgeräumt mit dem wissenschaftlichen Unfug, dass man den Eierkonsum möglichst reduzieren solle, weil Eier viel Cholesterin enthalten und Cholesterin koronare Herzkrankheit und Herzinfarkt begünstigt. In den USA und manchen anderen Ländern enthielten die Ernährungsrichtlinien solche Empfehlungen, Ernährungsampeln in Hotels platzierten eine rote Ampel beim Frühstücksei. Auch in Deutschland geisterte dieses wissenschaftliche Mythologem durch die Presse.

Eine kürzlich erschienene Studie [1] zeigt unmissverständlich: es ist ein Mythos. Die Studie ist aus drei Gründen bemerkenswert. 1. Sie stammt aus dem Department of Nutrition der Harvard School of Public Health, die über Dekaden dafür verantwortlich war, diesen Mythos von der bösen Wirkung des Cholesterins zu zementieren, eine Art öffentlicher Abbitte sozusagen. Vielleicht haben die Forscher es deswegen vorgezogen, das Ergebnis im British Medical Journal zu publizieren, also etwas abseits vom amerikanischen medizinischen Mainstream? 2. Es handelt sich nicht nur um eine der größten und längsten Kohortenstudien zum Thema. Sondern, 3. sie enthält auch eine Meta-Analyse aller bisher zum Thema durchgeführten Beobachtungsstudien.

Die Kohortenstudie umfasst insgesamt über 200000 Personen und mehr als 5 Millionen Personenjahre Beobachtung seit 1976. Ausgeschlossen wurden all diejenigen Menschen, bei denen zum Baseline-Zeitpunkt irgendeine chronische Krankheit vorlag. Der Eierkonsum wurde über einen Er-

\title{
Die Rehabilitierung des Eis
}

nährungsfragebogen erhoben, der alle 2-4 Jahre erneut abgefragt wurde, so dass wir von einem longitudinalen Datensatz ausgehen können. In einem logistischen Regressionsmodell, indem für sehr viele Lebensstilund Ernährungsparameter statistisch korrigiert wurde, zeigte sich überhaupt kein Zusammenhang zwischen kardiovaskulärer Erkrankung oder Herzinfarkt und Eierkonsum. Beim unkorrigierten Modell ist ein leichter linearer Zusammenhang erkennbar, der aber nicht einmal da zu einem statistisch signifikanten Einfluss der höchsten Konsumkategorie, ein Ei pro Tag oder mehr, auf Erkrankungsrisiko führt. Dieser lineare Zusammenhang verschwindet im korrigierten Modell komplett. Hier ist die Hazard Ratio sogar nicht signifikant unter Null, zeigt also einen leicht protektiven Effekt. Das liegt daran, dass mit dem Eierkonsum eben auch der Konsum manch anderer Lebensmittel verbunden ist, die vielleicht einen deutlicheren, auch nachvollziehbareren Effekt auf koronare Herzkrankheiten haben. Dieser Effekt wird im statistischen Modell korrigiert.

Die Meta-Analyse fasst 33 Kohorten zusammen und kommt zum klaren Ergebnis: Das Relative Risiko über alle Studien hinweg für Eierkonsum auf kardiovaskuläre Erkrankung ist 0.98. Wer mehr Eier isst, hat also ein um $2 \%$ gesenktes Risiko gegenüber denen, die keine Eier essen. Allerdings ist der Effekt nicht signifikant, denn das 95\%-Konfidenzintervall reicht von 0.93-1.03.

Es hat also 30 plus diese 3 Kohorten benötigt, um mit einem Vorurteil aufzuräumen, das zu allererst durch schlechte Forschung in die Welt gesetzt wurde - vergleiche mei- nen Kompassbeitrag «Unsere glykotoxische Ära geht zu Ende» [2]. Eine unselige Allianz aus wirtschaftlichen Interessen, in dem Fall der Zuckerindustrie, einigen Wissenschaftsmatadoren mit überblähtem Ego und zu viel Einfluss auf Politiker und einem sich selbst verstärkenden Zirkel aus Forschungsförderung mit der Notwendigkeit «richtige» Daten zu erzeugen hat diesen Mythos [3] in die Welt gesetzt und am Leben erhalten [4]. Er hat geschätzte $60 \mathrm{Mil}-$ lionen Dollar an Forschungsgeldern verbrannt, bis wir dort angelangt sind, wo wir vorher immer schon waren: Bei der Erkenntnis dass Eier, mindestens für die, die sie mögen, ein wertvolles Lebensmittel sind, weil sie viel Lecithin, Phospholipide, Lutein und noch ein paar andere wertvolle Inhaltsstoffe enthalten und dass sie auf keinen Fall schädlich sind. Manche nennen so etwas Fortschritt. Ich finde, mit ein bisschen Hirn hätte man sich diese Art von Fortschritt sparen können, denn wir wissen aus der Physiologie schon lange, dass der Körper maximal 5\% des Cholesterinhaushaltes über die Nahrung reguliert [5].

Das Ei ist also wieder auferstanden und wir können getrost täglich Ostern feiern, mit einem, vielleicht auch 2 Eiern.

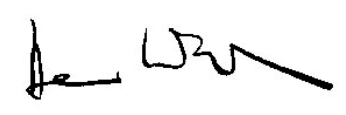

Prof. Dr. Dr. phil. Harald Walach

\section{Literatur}

Die Literatur ist unter https://www.karger.com/ Article/FullText/516544 abrufbar. 